\title{
Price Discovery in Cryptocurrency Markets
}

\author{
By Igor Makarov and Antoinette Schoar*
}

Over the last two years cryptocurrencies have gone through what appears to be a classic asset bubble: After a dramatic run-up in prices during 2017, the price of Bitcoin fell by more than 80 percent in the first half of 2018 . Similar dynamics occurred in nearly all other cryptocurrencies. Economists typically define a bubble as a period where the price of an asset diverges from its "fundamentals." Those fundamentals are usually valued based on the income stream that can be earned from an asset over time, say a company's cash flow, or the rent from a property. But Bitcoin does not pay dividends like shares do or rent like real estate, nor is it backed by a national economy as fiat currencies are. The ultimate value of Bitcoin then is based on the likelihood of its adoption as a global currency and on its success as a payment system. As evident from any chatroom about cryptocurrencies, this market has attracted a diverse set of investors around the world including many diehard believers, speculators, as well as many skeptics.

In this paper we study how price discovery happens in the Bitcoin market. We build on our earlier paper (Makarov and Schoar 2018), which documents that cryptocurrency markets around the world are partially segmented and experience extended periods where prices deviate substantially from the law of one price. These price deviations seem to persist due to the slow-moving capital (see Duffie 2010) and capital controls in many countries that hinder the efficient flow of existing arbitrage capital across exchanges.

\footnotetext{
* Makarov: London School of Economics, Houghton Street, London, WC2A 2AE (email: i.makarov@1se.ac.uk); Schoar: Sloan School of Management, MIT, 100 Main Street, Cambridge, MA 02139 (email: aschoar@mit.edu). We thank Jonathan Berk for very thoughtful comments on our research.

${ }^{\dagger}$ Go to https://doi.org/10.1257/pandp.20191020 to visit the article page for additional materials and author disclosure statement(s).
}

Our earlier results suggest that the marginal investor outside the United States and Europe is willing to pay more for Bitcoin in response to the boom in crypto prices. We conjecture that investors in countries with poorly functioning financial institutions or tighter capital controls might value Bitcoin more highly, since they would benefit more from the adoption of cryptocurrencies. Thus, these countries have higher sensitivity of Bitcoin prices to news about the potential adoption of Bitcoin (or any positive shock to sentiment).

In this paper we ask which markets drive Bitcoin prices. Does the greater exuberance for cryptocurrencies outside the United States affect prices only on these local markets or do they have an effect on price formation on global cryptocurrency markets? We show that there is significant heterogeneity in where price formation happens across exchanges and time. In times where markets are more integrated, and price deviations are small, shocks to prices on all exchanges contribute to price discovery. However, when markets become segmented, those exchanges that have large arbitrage spreads relative to the US price become much less important for price discovery. In other words, the impact of price movements in the most exuberant markets, say Korea during the Kimchi premium, only have a minimal effect on the global price of Bitcoin.

\section{Background}

In our earlier work we used tick level trading data from 34 exchanges across 19 countries. For a description of the data, see Makarov and Schoar (2018). We show that there are significant barriers to arbitrage between regions and, to a lesser extent, even between exchanges in the same country. There are large and recurring deviations in Bitcoin prices across exchanges that open up across different exchanges and often persist for several hours, and, in some instances, days and weeks. These price deviations are mainly driven by frictions across countries (or 
regions) rather than within the same country. We document that the large deviations exist even between regions with the most liquid exchanges, such as the United States, Japan, Korea and, to a lesser extent, Europe.

In further support of the idea that capital controls play an important role, we find that arbitrage spreads are an order of magnitude smaller between cryptocurrencies (say Bitcoin to Ethereum or to Ripple) on the exact same exchanges where we see big and persistent arbitrage spreads relative to fiat currencies. Since the main difference between fiat and cryptocurrencies is the inability to enforce capital controls, our findings suggest that such controls contribute to the large arbitrage spreads we find across regions.

In addition, we show that price deviations occur during periods of a particularly quick appreciation of Bitcoin prices. These periods also coincide with the times when there is a particularly strong increase in demand for Bitcoin worldwide. We show that the countries that on average have a higher premium over the US Bitcoin price are also those with a higher sensitivity of the local price to the world market price of Bitcoin. So these countries respond more strongly by widening arbitrage deviations during times when buying pressure goes up in the United States.

\section{Price Discovery}

To analyze on which markets new information is incorporated into the price of Bitcoin we follow Hasbrouck (1995). This approach assumes that Bitcoin prices across the world, $p_{t}=\left[p_{1 t}, \ldots, p_{n t}\right]$, are cointegrated and their dynamics are represented by vector error correction model:

$\Delta p_{t}=\gamma B p_{t-1}+\varphi_{1} \Delta p_{t-1}+\cdots+\varphi_{k} \Delta p_{t-k}+\varepsilon_{t}$,

where $B=\left[\begin{array}{cc}-1 & \\ \vdots & I_{n-1} \\ -1 & \end{array}\right], E \varepsilon_{t}=0$, and $E \varepsilon_{t} \varepsilon_{s}=\Omega$

for $t=s$ and 0 otherwise. The first term on the right-hand side is the error correction term.

Cointegration stipulates that while two price series, say the price of Bitcoin in Korea and the United States, might not be stationary, their difference is stationary because of no-arbitrage conditions between the two markets. The
$B p_{t-1}$ product defines the cointegrating vectors, which we specify without loss of generality as $p_{2 t}-p_{1 t}, p_{3 t}-p_{1 t}, \ldots, p_{n t}-p_{1 t}$.

Cointegration implies that there is an unobservable "efficient" price which is common to all markets. Price discovery in this framework refers to innovation in the efficient price. A market's contribution to price discovery is its proportion of the variance in the efficient price innovations that can be attributed to this market.

We use data from the five most prominent Bitcoin markets: United States, Europe, Japan, Korea, and Tether. Tether is a cryptocurrency that is supposed to be pegged one-for-one to the US dollar. The goal is to create a stable cryptocurrency that serves as a digital dollar and facilitates trading in crypto exchanges by avoiding the frictions associated with fiat currency transactions. At the peak of the Bitcoin boom almost 50 percent of trading in Bitcoin was on Tether based exchanges (Makarov and Schoar 2018). In each market we use transaction prices from the most liquid exchange there. These are Coinbase (United States), Bitstamp (Europe), Bitfinex (Tether), BitFlyer (Japan), and Bithumb (Korea). In our base specification, we use a time interval of one second and past lags for up to six hours. To deal with a large number of parameters we constrain the coefficients $\varphi_{k}$ to be the same at progressively higher resolutions. The coefficients $\varphi_{1}, \ldots, \varphi_{5}$ are unrestricted, $\varphi_{6}=\cdots=\varphi_{10}, \quad \varphi_{11}=\cdots$ $=\varphi_{60}, \varphi_{61}=\cdots=\varphi_{300}, \varphi_{301}=\cdots=\varphi_{3,600}$, and $\varphi_{3,601}=\cdots=\varphi_{21,600}$. The analysis is carried over the period from September 2017 to February 2018.

Table 1 shows the results from this analysis. The rows refer to particular markets and the columns are the loadings of the long-run effect of a shock in a particular market on the efficient price. This analysis is meaningful since we find that these shocks across markets are independent of each other, which means the variance-covariance matrix is diagonal. We constrain these shocks to have the same variance, for ease of interpretation. In theory, if price deviations were short lived, each row should have the same coefficient, since these are loading on the efficient (common) price. But as we showed in our earlier paper, price deviations especially in Japan and Korea can sometimes take more than a day to converge. So even over the horizon of the six hour lags we used in this 
Table 1 -Contribution to Price Discovery

\begin{tabular}{lccccc}
\hline \hline & Coinbase (US) & Coinbase (Euro) & BitFlyer & Bitfinex & Bithumb \\
\hline Coinbase (US) & 0.28 & 0.08 & 0.21 & 0.48 & 0.09 \\
Coinbase (Euro) & 0.24 & 0.11 & 0.20 & 0.50 & 0.09 \\
BitFlyer & 0.21 & 0.08 & 0.39 & 0.43 & 0.09 \\
Bitfinex & 0.21 & 0.08 & 0.20 & 0.54 & 0.09 \\
Bithumb & 0.18 & 0.08 & 0.19 & 0.42 & 0.35 \\
\hline
\end{tabular}

Notes: The analysis in this table follows Hasbrouck (1995). The rows refer to particular markets and the columns are the loadings of the long-run effect of a shock in a particular market on the efficient price. Shocks across markets are independent of each other, which means the variance-covariance matrix is diagonal. We constrain these shocks to have the same variance.

Source: Author calculations. Data from Kaiko for the period from September 2017 to January 15, 2018.

analysis, the deviations in prices might not have fully converged.

From Table 1 we see that the markets that are most important for price discovery are Bitfinex (Tether) and to a slightly lesser extent Coinbase (United States) and BitFlyer (Japan). These are three of the largest and most liquid cryptocurrency markets. Many observers have conjectured that especially Bitfinex plays a significant role in price formation since the digital nature of Tether might allow a large and diverse set of traders to participate in this market who might be constrained from trading in other markets due to regulations on fiat currencies. In fact, some concerns have been raised that Tether has even been used to push up the price of Bitcoin. In contrast, the contribution of Korea and Europe are much smaller. Finally, when looking at BitFlyer (Japan) and Bithumb (Korea) we see that while the impact on other exchanges is moderate, the impact of these exchanges on itself is large. In comparison, this effect is much smaller for the United States, Europe, and Tether based markets. This result again confirms that the degree of integration with the global Bitcoin market is lower for Korea and Japan.

We then repeat the analysis for the two subperiods from September 2017 to December 15, 2017 and the second from December 15, 2017 to January 15, 2018 (not reported here). The latter period was the height of the Bitcoin bubble. Most of the results for the two sub-periods are qualitatively unchanged from those in Table 1, with one interesting exception: during the latter period the contribution of the Korean market (Bithumb) to price discovery fell by more than half. This suggests that during the height of the boom, a market that saw one of the biggest deviations from the global market price (often called the "Kimchi premium") did not significantly contribute to the run-up in overall prices. In other words, the spillover effects from some of the most exuberant markets onto the worldwide Bitcoin price was limited.

\section{Conclusion}

This paper provides a first look at the role that trading in different markets plays for the global price of Bitcoin. We document significant heterogeneity in where price discovery happens and how these contributions changed during the height of the Bitcoin boom compared to more stable time periods. Especially US, Japan, and Tether-based markets appear to play a central role for price formation. Going forward it will be of great interest to understand how investors in these markets form expectations about the value of cryptocurrencies, what type of information they respond to, and how they decide which markets to trade in.

\section{REFERENCES}

Duffie, Darrell. 2010. "Presidential Address: Asset Price Dynamics with Slow-Moving Capital." Journal of Finance 65 (4): 1237-67.

Hasbrouck, Joel. 1995. "One Security, Many Markets: Determining the Contributions to Price Discovery." Journal of Finance 50 (4): 1175-99.

Makarov, Igor, and Antoinette Schoar. 2018. "Trading and Arbitrage in Cryptocurrency Markets." Unpublished. 